\title{
超长线列红外探测器与制冷机耦合的柔性冷链发展现状
}

\author{
邓 蔚, 孙鸿生, 朱颖峰, 徐冬梅, 李 再, 黄一涁 \\ (昆明物理研究所, 云南 昆明 650223)
}

\begin{abstract}
摘要: 随着航天技术对更大视场、更高分辨率的需求, 线列红外探测器规模越来越大, 传统的制冷机 与红外探测器单点耦合方式, 已经无法满足超长线列红外探测器芯片温度均匀性的要求。结合国内外 多家研究单位的设计及试验情况, 对各类柔性冷链进行了对比分析, 从热学性能及力学性能出发, 总 结了各类柔性冷链设计的特点及其适用性。
\end{abstract}

关键词: 超长线列红外探测器; 制冷机; 耦合; 柔性冷链

中图分类号：TN215 文献标识码：A 文章编号：1001-8891(2020)01-0010-09

\section{Development Status of the Flexible Thermal Link Coupling Between Cryocooler and Long Linear Infrared Detector}

\author{
DENG Wei， SUN Hongsheng, ZHU Yingfeng, XU Dongmei， LI Ran, HUANG Yibin
}

(Kunming Institute of Physics, Kunming 650223, China)

\begin{abstract}
With the demand of from space technology for a large field of view and higher resolution, the scale of line infrared detectors is increasing. Single point coupling mode fails to meet the temperature uniformity requirement of long linear infrared detector chips. This study compares and analyzes various types of flexible thermal links, based on the design and the testing outcomes from many domestic and international research institutes. The characteristics and applicability of the various flexible thermal link designs, in terms of thermal and mechanical properties, are summarized.
\end{abstract}

Key words: long linear infrared detector, cryocooler, coupling, flexible thermal link

\section{0 引言}

超长线列红外探测器组件目前已经达到几千元 的规模, 线列长度达到 $200 \mathrm{~mm}$, 广泛使用在红外遥 感应用中。由于红外探测器常需在低温下进行工作, 而机械制冷具有结构紧凑、体积小、重量轻、制冷量 大、制冷时间短、制冷温度可控范围大等优点, 目前 该类探测器在应用中大多采用机械制冷的方式。随着 红外探测器面阵规模的变大, 小型杜瓦单点连接的耦 合方式已经无法满足要求, 因此对超长线列探测器杜 瓦与制冷机耦合方式提出新的需求。

本文从超长线列红外探测器与制冷机的耦合方 式出发, 综合国内外的柔性冷链研究及发展现状, 对 冷链设计及制作工艺进行分析。

\section{1 超长线列红外探测器与制冷机耦合的研究 现状}

超长线列红外探测器与制冷机的集成式耦合有
两种实现的技术方法: 一种为钎焊式, 即制冷机的冷 指直接通过钎焊与冷板焊接在一起, 考虑到焊接过程 中的热匹配性, 冷板需采用膨胀系数较大的材料, 如 钿铜, 但膨胀系数大的冷板将大大增加冷头设计的困 难, 需在冷头上设计复杂的应力卸载结构, 并且因是 单点冷源, 探测器焦平面上的温度均匀性很难保证, 如图 1 为 “高分 5 号” 卫星长线列红外探测器组件结 构图 ${ }^{[1]}$, 采用的就是钎焊式的耦合结构; 第二种为冷 链式, 制冷机冷指通过冷链与冷板进行连接, 因制冷 机不直接与冷板相接, 其冷头不需要复杂的应力卸载 结构, 同时可根据焦平面上的温度均匀性需求, 来灵 活调整热量传输结构, 并且可以通过隔振设计来降低 制冷机传导至焦平面的振动, 如图 2 为上海技物所直 线脉管集成耦合杜瓦结构 ${ }^{[2]}$, 采用的就是冷链式的耦 合结构。对于超长线列红外探测器, 为保证探测器的 温度均匀性, 应采用冷链式的耦合方法, 因此冷链尤 其是柔性冷链研究是超长线列红外探测器工程化的 重点。 

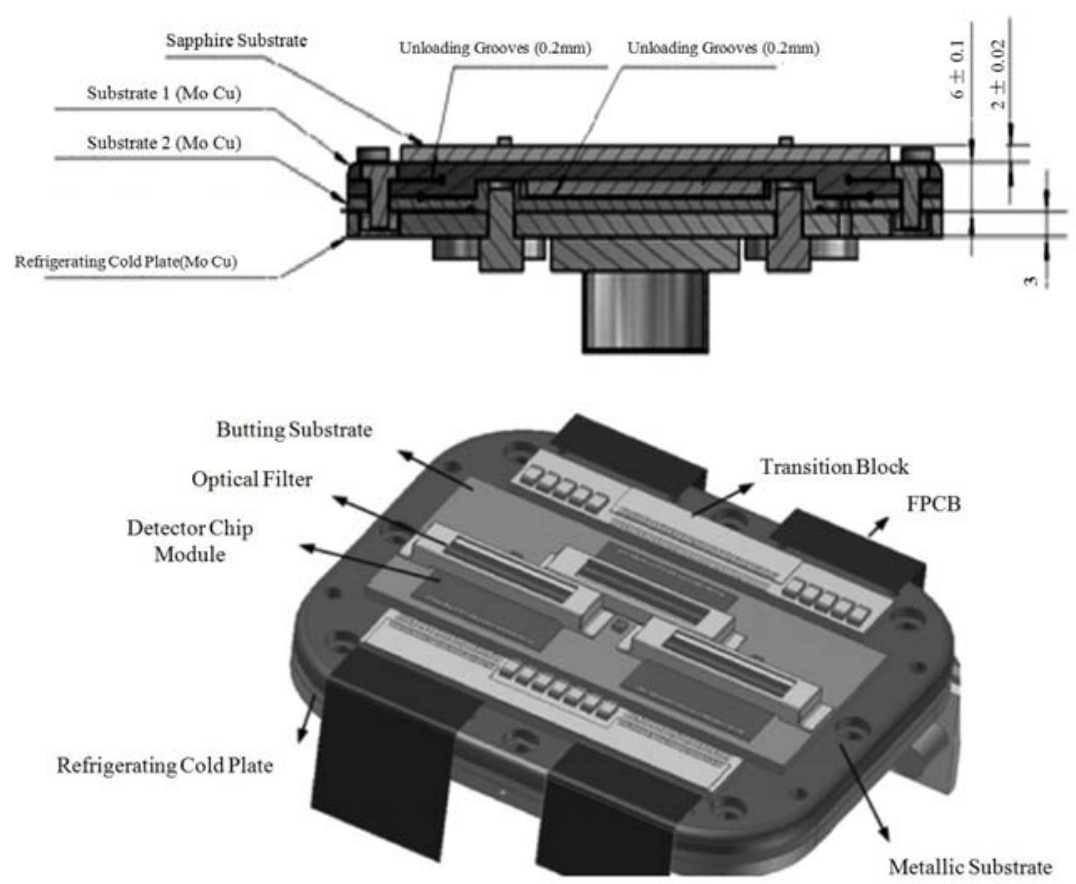

图 1 “高分 5 号”卫星长线列红外探测器组件应力卸载结构图 (上图) 和焦平面结构图 (下图)

Fig.1 Stress unloading structure (up) and focal plane structure (down) of GF-5 satellite linear infrared detector components

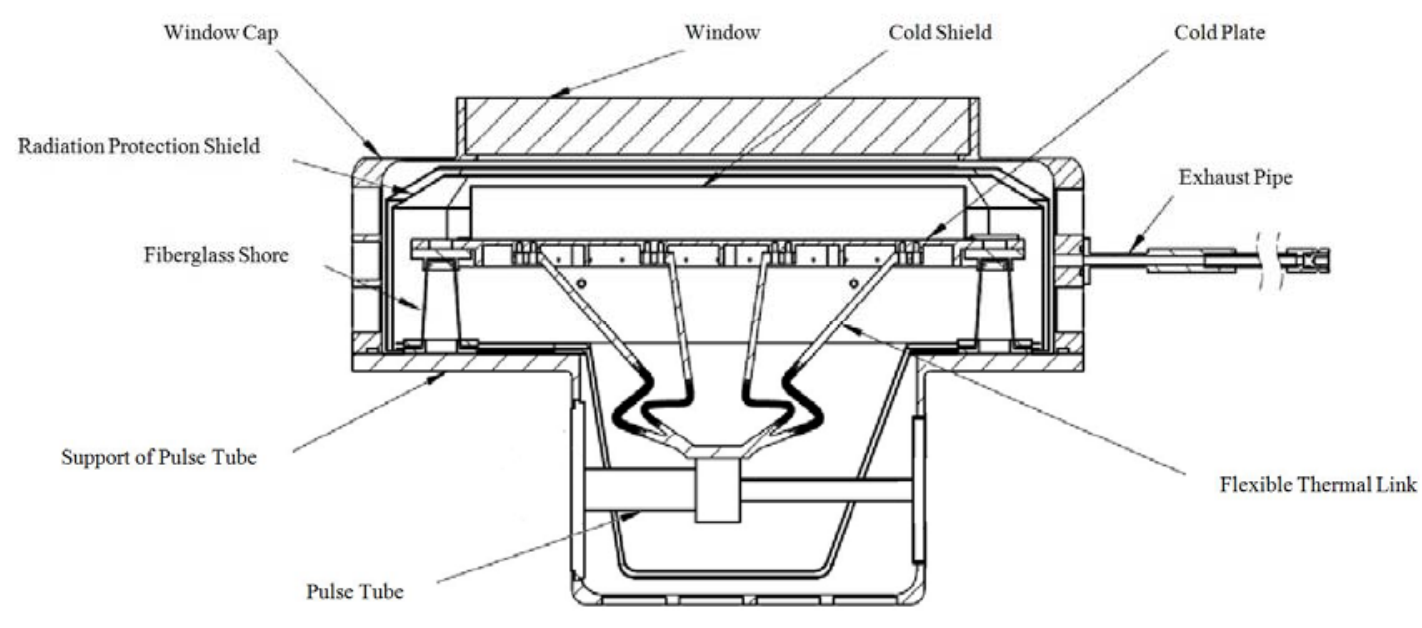

图 2 上海技物所长线列红外探测器杜瓦结构图

Fig.2 Dewar structure of linear infrared detector designed by Shanghai Institute of Technical Physics

\section{2 柔性冷链的国内外研究现状}

\section{1 柔性冷链的国外研究现状}

\subsection{1 柔性冷链的结构}

柔性冷链作为冷量传输渠道, 能够更加灵活地分 配冷量、隔绝振动, 常应用于各种空间项目中。对于 柔性冷链, 国外已经做了大量的研究, 并且已经有了 成熟的冷链设计、加工方法和检测手段, 柔性冷链按 形状结构分类, 可分为链状、片状、丝状和部分异形 冷链。各种类型的冷链结构都是通过将冷链分割成多
层薄片或多根导热丝的方法, 来减小冷链整体结构的 惯性矩来获得结构的低刚度。但不同结构具有不同的 应用领域。

链状冷链采用丝状材料编织而成的链状结构作 为冷链的柔性部分, 这种结构的冷链具有 3 个方向的 自由度, 能够在 3 个方向进行自由运动, 但因链状结 构制作工艺的问题, 链状冷链比较难保持高清洁度, 容易在使用过程中产生多余物，同时因这种结构表面 积大, 易吸附气体, 故链状冷链不适在高清洁度和高 真空度的环境下使用。 
如图 3 所示, 目前链状冷链主要有两种: 一种是 采用高纯度无氧铜丝编织而成的铜链, 铜链能与铜热 接头制成一体式冷链, 并且制作工艺成熟, 广泛应用 在空间项目中, 如表 1 所示为 TAI (Technology Applications Inc. ) 公司统计的使用过链状铜冷链的空 间项目, 在空间项目中链状冷链最常使用的方式是与 刚性槽道热管配合形成完整的热量传输系统, 如图 4
为链状铜冷链的装配图; 一种是采用捆扎的碳纤维管 束作为柔性部分, 配合铜或铝热接头的分体式柔性冷 链, 相比无氧铜, 碳纤维在 $100 \mathrm{~K}$ 温度以上具有更高 的热导率, 更小的密度和刚度, 是能够替代链状铜冷 链的新型链状冷链的制作材料, 目前也已经在空间项 目中大规模使用, 如表 2 所示为 TAI 公司统计的使用 过碳纤维管束冷链的空间项目。
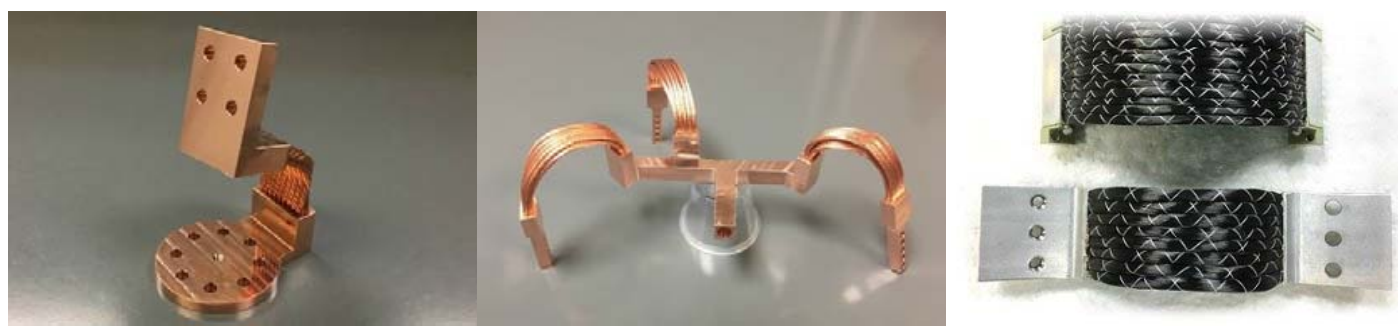

图 3 链状柔性冷链: 无氧铜链状柔性冷链实物图 (左图和中图) 、碳纤维管束链状柔性冷链实物图 (右图)

Fig.3 Chain-shaped flexible thermal link: flexible thermal link of oxygen-free copper (left and middle picture), flexible thermal link of carbon fiber bundle (right)

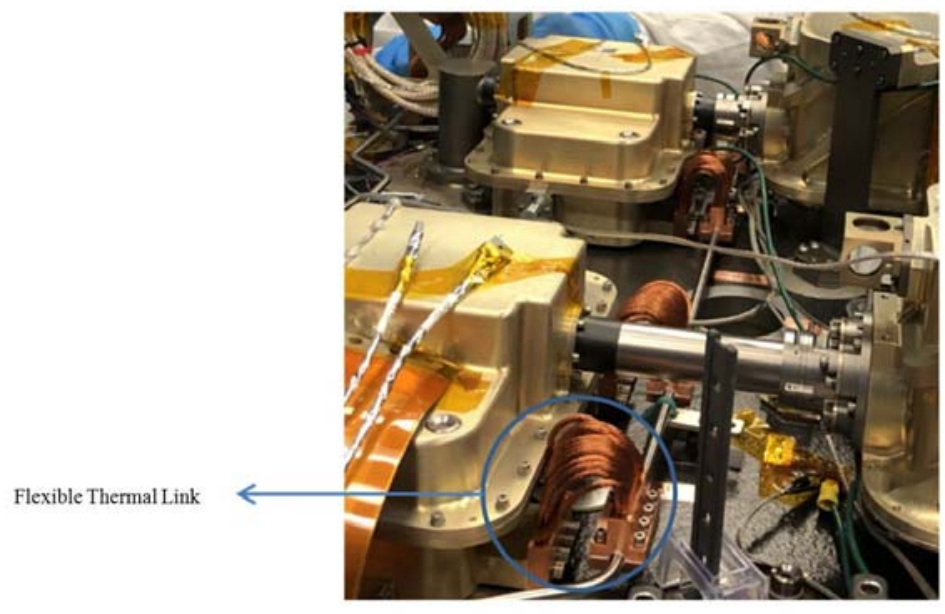

图 4 链状柔性冷链装配图

Fig.4 Assembly structure of chainlike flexible thermal link

表 1 采用铜冷链热传导的空间项目 Table 1 Space items using copper thermal link

\begin{tabular}{lc}
\hline Project name & Launch time \\
\hline Lockheed Martin/NOAA's GOES-R & 2016.11 \\
NASA's OSIRIS REx & 2016.9 \\
Numerous Department of Defense Satellites & $2016-2018$ \\
NOAA's GOES-S/T/U & $2017-2018$ \\
NASA JPL's GRACE-FO & 2018 \\
Orbital ATK's Cignus OA-9 Spacecraft & 2018.5 \\
NASA's GEDI Sensor(on board the ISS) & 2018.11 \\
Eumetsat's METEOSAT & 2020 \\
NASA JPL's Deep Space Atomic Clock(DSAC) & 2019.3 \\
ESA's Solar Orbiter & 2019.2 \\
\hline
\end{tabular}


表 2 采用碳纤维管柔性冷链热传导的空间项目

\begin{tabular}{lc}
\hline Project name & Launch time \\
\hline NASA's ORION Spacecraft & 2015.11 \\
JAXA's ASTRO-H(Hitomi) & 2016.2 \\
NASA JPL's GRACE-FO & 2018.5 \\
USAF's GPS III & 2018.11 \\
Numerous Department of Defense Satellite Programs & $2016-2018$ \\
ESA\&NASA's Spectral Imaging of the Coronal Environment(SPICE) instrument(on Solar Orbiter) & 2020.2 \\
Boeing's CST-100 Commercial Crew Vehicle & 2019.7 \\
DLR's EnMAP & 2020 \\
Ball Aerospace's GEMS UV-VIS Spectrometer(GEO-KOMP SAT-2B Satellite) & 2019 \\
NASA's IXPE Mission & 2021 \\
\hline
\end{tabular}

1996 年荷兰的 S. Dolce 针对碳纤维的高热导率, 使用二百万根直径为 $0.01 \mathrm{~mm}$ 的碳纤维管束制作了一 种柔性冷链, 该冷链采用了 “C” 形结构来减小 $Z$ 方 向的刚度, 在碳纤维管束的上下两端使用铝块压紧, 冷链外围用聚酯薄膜包裹, 来减小辐射漏热的同时防 止碳碎屑飞出, 其结构如图 5 所示 ${ }^{[3]}$ 。在后续试验中, 该冷链包括上下铝块及耦合处其总体的热导率能够 达到 $300 \mathrm{~K}$ 下 $1.3 \mathrm{~W} / \mathrm{K}, 100 \mathrm{~K}$ 下 $0.7 \mathrm{~W} / \mathrm{K}$; 该冷链 $X$, $Y$ 轴上的刚度在 $300 \mathrm{~K}$ 温度下空气中为 $0.75 \mathrm{~N} / \mathrm{mm}$,
Table 2 Space items using carbon fiber bundle thermal link

在 $100 \mathrm{~K}$ 温度下真空中为 $2.5 \mathrm{~N} / \mathrm{mm}$, 其 $Z$ 轴的刚度在 $300 \mathrm{~K}$ 温度为 $3.5 \mathrm{~N} / \mathrm{mm}$ 。

因在高真空或高清洁的环境内, 链状冷链会污染 环境不能使用, 需考虑其他方式的柔性冷链结构, 而 以铜箔、铝䈃或柔性石墨为主体的片状柔性冷链能够 比较好地满足要求, 片状柔性冷链能够较好地清洁, 并且表面积相对较小, 尤其是只需要考虑两个方向的 自由度时, 片状柔性冷链是最好的选择, 常被使用于 各类探测器中, 如图 6 所示。
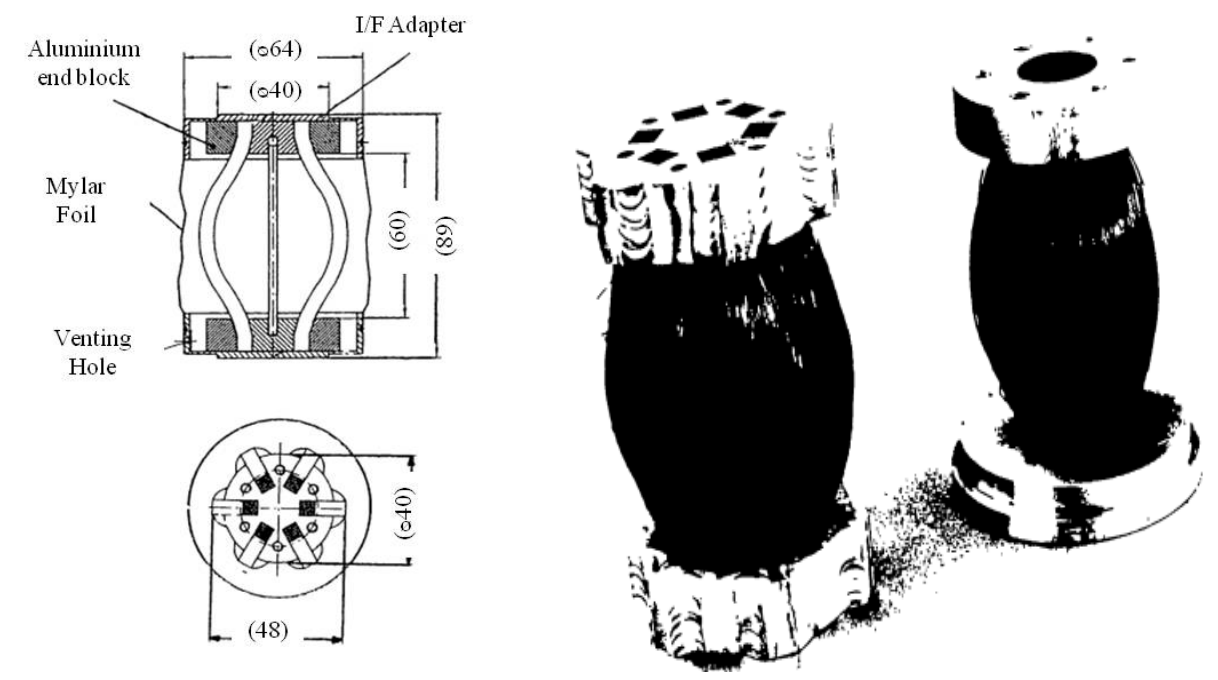

图 5 碳纤维管柔性冷链结构示意图 (左图) 和实物图 (右图)

Fig.5 Structure sketch(left) and physical map(right) of carbon fiber bundle thermal link
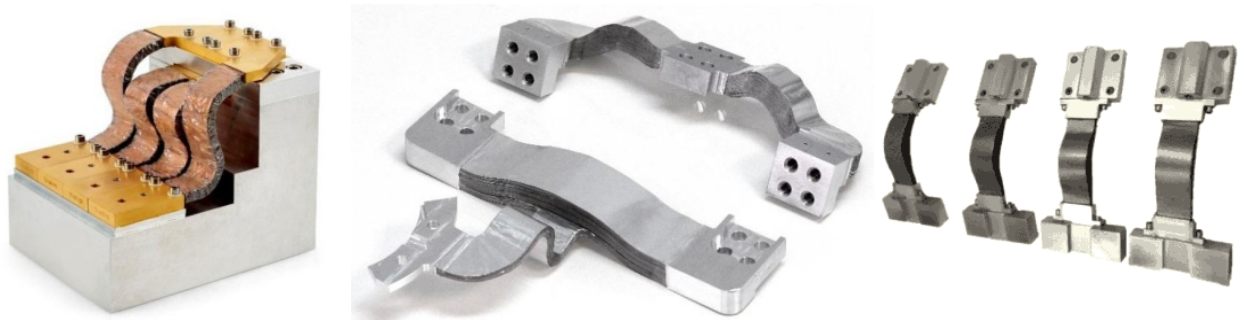

图 6 片状柔性冷链: 铜片状冷链 (左图) 、铝片状冷链（中图）、石墨片状冷链（右图）

Fig.6 Foil thermal straps: copper foil thermal straps(left), aluminum foil thermal straps(middle), graphite foil thermal straps(right) 
1997 年美国航天局发射的 MIDAS (The Materials In Devices As Superconductors)中采用了 11 片 $0.1 \mathrm{~mm}$ 厚的铜薄片组成的柔性冷链, 来连接高温超导材料与 制冷机冷头, 该冷链的两端采用螺钉固定紧压, 在固 定处为了减小接触热阻在接触点处垫入了铟片, 该冷 链能在隔绝制冷机振动同时具有高的热导率, 最终使 高温超导材料与制冷机冷头之间的温差小于 $2 \mathrm{~K}^{[4]}$, 结构如图 7 所示。

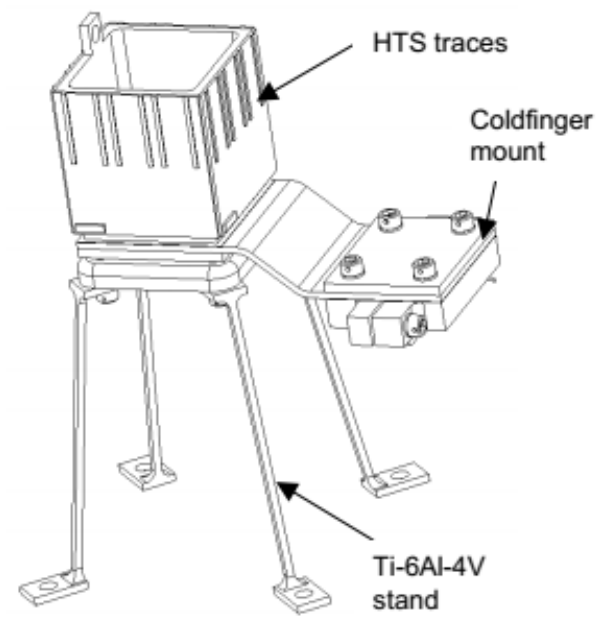

图 7 MIDAS 传热结构图

Fig.7 MIDAS heat transfer structure

美国国家海洋和大气局发射 GEOS (Geostationary Operational Environmental Satellite) ABS (Advanced Baseline Sounder ${ }^{[5]}$ 中的杜瓦组件中由于冷头位置距 离探测器较远, 因而制冷机冷头通过柔性冷链连接到 焦平面器件的安装面, 柔性冷链由多层铝片组成, 在 低温下仍可保持一定的柔性, 可以消除冷链低温收缩 所产生的应力, 采用铝作为冷链材料可以在低温下获 得与铜接近的热导, 重量上比铜要轻, 有利于组件的 轻量化和振动条件下的可靠性, 结构如图 8 所示。

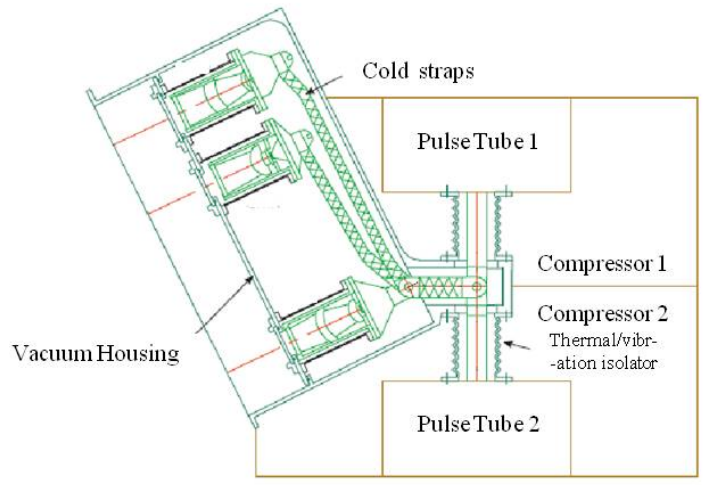

图 8 GEOS 杜瓦组件结构图 Fig.8 GEOS dewar structure 当需考虑 3 个方向自由度, 且应用于高真空度, 高清洁度的环境中时, 可采用金属丝进行连接。1996 年美国航天局发射的 EOS（Earth Observing System）
AIRS (Atmospheric Infrared Sounder) ${ }^{[6]}$ 中的杜瓦组件 陶瓷基板通过局部柔性冷链与冷头连接, 局部柔性冷 链与基板的连接端由铝制成，与冷头的连接端设计为 由多根金属丝连接的两块金属板，金属丝实现了冷链 的局部柔性，两个连接端之间为镀金的宝石棒，宝石 在该杜瓦的工作温度 $60 \mathrm{~K}$ 具有很高的热导率，可以 有效地降低冷链的导热温差, 宝石棒通过焊接与两连 接端连接, 采用此局部柔性冷链设计可以使得低温下 产生的热应力通过冷链弹性部分得以释放, 同时可以 隔离探测器与制冷机冷头之间的振动耦合, 结构如图 9 所示。

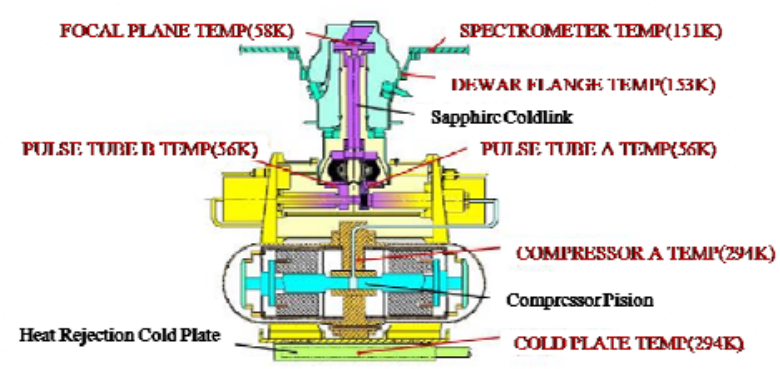

图 9 AIRS 杜瓦组件结构图

Fig.9 AIRS dewar structure

除了以上较为常规的冷链结构, 也有一些较为特 殊的柔性冷链结构。日本的 Masahiro Sugimoto 为了 保持 SIS (superconducting-insulator-superconducting tunnel junction） ${ }^{[7]}$ 系统温度的同时不受机械制冷机振 动的影响, 采用了如图 10 所示柔性的连接方式。该 连接整体采用无氧铜材料, 支撑部分厚度为 $0.3 \mathrm{~mm}$, 并沿轴方向开了 120 条坚槽从而来减小整体的刚度, 该连接在外圈用尼龙绳固定压紧, 同时通过尼龙与铜 之间的热膨胀系数的差异, 温度越低尼龙绳固定压紧 的力越大。通过测试该耦合结构在 $80 \mathrm{~K}$ 温度下热导 率达到 $4 \mathrm{~W} / \mathrm{K}, 12 \mathrm{~K}$ 温度下热导率达到 $2.3 \mathrm{~W} / \mathrm{K}$, 同 时该耦合结构能将由制冷机带来的振幅由 $30 \mu \mathrm{m}$ 减至 $6 \mu \mathrm{m}$ 。

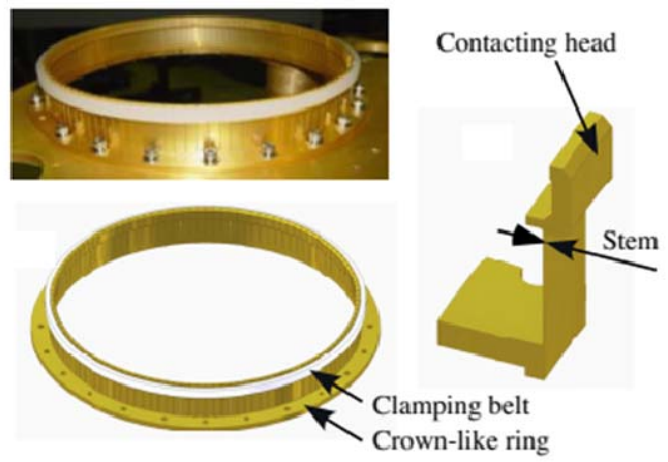

图 10 SIS 系统中传热的柔性结构

Fig.10 The flexible structure of heat-transfer in SIS systems 


\subsection{2 柔性冷链的材料}

柔性冷链采用的材料有 3 种大类: 铜、铝及石墨 材料。如图 11 为这几种材料在不同温度下的热导率 图, 在红外探测器使用的温度区 $80 \mathrm{~K} \sim 100 \mathrm{~K}$ 左右, 无氧铜的热导率最大, 柔性石墨材料其次, 1100 号铝 金属热导率最低。同时考虑冷链本身的重量, 以热导 率比密度值作为衡量的标准, 结果如表 3, 在这 3 种 材料中, 热导率比密度值石墨最大, 铝其次, 铜最小。 故在空间应用中, 相同质量下, 冷链使用石墨传热效 果最佳, 铝其次, 铜最差。除材料本身的性质之外, 柔性冷链中制作工艺的选择对冷链整体的热导率及 可靠性至关重要。国外对这些方面已经做过大量的工 作。AS (Absolut System) 公司的 T. Trollier 对不同纯 度的铝金属的热导率进行了测量, 测量结果如表 4 和 图 12 所示 (图中 4N、5N、6N 代表铝金属的纯度的 高低, 如 4N 表示铝金属的纯度为 $99.99 \%$ ）, 当铝金 属中铝的纯度越大时, 其热导率越高, 纯度为 $99.999 \%$ 铝金属低温下的热导率是纯度为 $99 \%$ 铝金属热导率 的 1 2 倍。同时, $\mathrm{T}$. Trollier 对铝簿与铝热接头的焊 接工艺、铜簿与铜接头的焊接工艺和铜䈃与铝接头的 焊接工艺进行了实验和测量, 结果如表 5 所示。结果 表明, 铝䈃与铝热接头的焊接采用离子束焊接效果最 好, 铜箔与铜接头的焊接采用压焊及离子束焊接的效 果最好, 铜䈃与铝接头的焊接采用钎焊的效果最好, 这些焊接都能够达到焊接处牢固且无接触热阻产生 ${ }^{[8]}$ 。

石墨材料具有高热导率、良好的力学性能和低密
度, 是制作柔性冷链最理想的材料, 如图 13 所示为 部分柔性石墨冷链的示意图。对于石墨柔性冷链, 主 要的难点是石墨柔性部分和刚性部分连接的可靠性, 以及如何解决石墨材料因各向异性的热导率带来的 温度梯度问题。

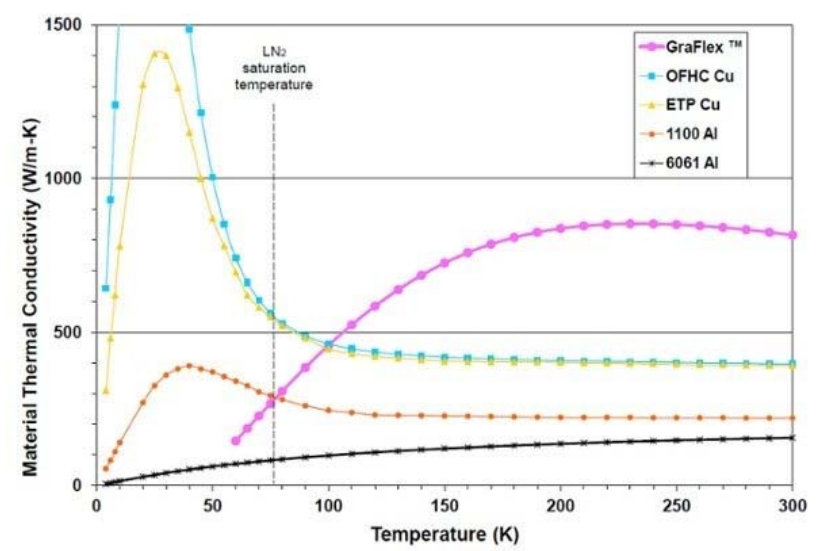

图 11 不同温度下几种高传热材料的热导率

Fig.11 The thermal conductivity of several materials with high-transfer performance at different temperatures

美国的 Mark J. Montesano 设计了一种以 APG (annealed pyrolytic graphite) 为主体, 使用铝薄片进 行封装的复合材料制造的柔性冷链 ${ }^{[9]}$, 在铝薄片和 APG 之间通过连接剂进行粘接, 通过调整铝薄片、 APG 及连接剂的厚度, 该复合材料最高能够达到 1105 $\mathrm{W} / \mathrm{mK}$ 的热导率, 结构如图 14 所示。

\section{表 3 铜、铝和石墨材料部分参数对比表}

Table 3 Comparison of partial parameters of copper, aluminum and graphite materials

\begin{tabular}{lccc}
\hline & Thermal Conductivity under $80 \mathrm{~K} /(\mathrm{W} / \mathrm{m} \cdot \mathrm{K})$ & Density $/\left(\mathrm{g} / \mathrm{cm}^{3}\right)$ & Thermal conductivity/Density \\
\hline OFHC Cu & 558 & 8.9 & 62.7 \\
$1100 \mathrm{Al}$ & 283 & 2.7 & 104.8 \\
GraFlex & 300 & 1.8 & 166.7 \\
\hline
\end{tabular}

表 4 纯度不同铝金属与无氧铜的热导率

Table 4 Thermal conductivities of aluminum with different purities and copper

\begin{tabular}{llllll}
\hline \multicolumn{5}{c}{ Thermal conductivity $(\mathrm{W} / \mathrm{m} \cdot \mathrm{K})$} \\
\hline \multicolumn{5}{c}{ Aluminum grade } \\
$T / \mathrm{K}$ & 1100 & 1050 & $4 \mathrm{~N}$ & $5 \mathrm{~N}$ & OFHC \\
50 & 369 & 425 & 948 & 1087 & 1173 \\
60 & 338 & 389 & 645 & 695 & 816 \\
70 & 308 & 354 & 484 & 507 & 646 \\
80 & 283 & 326 & 390 & 401 & 558 \\
Purity \% & 99 & 99.50 & 99.990 & 99.999 & 99.99 \\
\hline
\end{tabular}

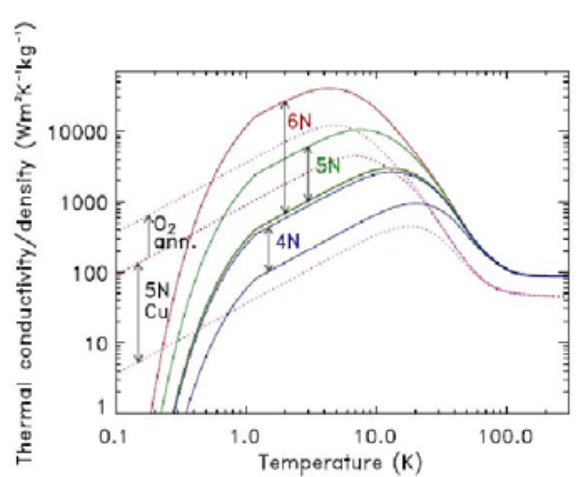

图 12 铝金属和无氧铜的热导率对比图

Fig.12 Comparison of thermal conductivity between aluminum and copper 


\section{表 5 不同金属管与热接头间焊接工艺研究}

Table 5 Feasibility study of assembling processes according to the foils and fittings materials

\begin{tabular}{|c|c|c|c|}
\hline $\begin{array}{l}\text { Processes available } \\
\text { for foil assembly }\end{array}$ & $\begin{array}{l}\text { Aluminum foils and } \\
\text { aluminum fittings }\end{array}$ & $\begin{array}{l}\text { Copper foils and copper } \\
\text { fittings }\end{array}$ & $\begin{array}{l}\text { Copper foils and } \\
\text { aluminum fittings }\end{array}$ \\
\hline TIG/MIG & $\begin{array}{l}\text { Good } \\
\text { but not critical on foils }\end{array}$ & Good & Not feasible \\
\hline Press-welding & Not feasible & $\begin{array}{l}\text { Very good } \\
\text { no thermal resistance } \\
\text { between foils }\end{array}$ & Not feasible \\
\hline Diffusion welding & $\begin{array}{l}\text { Feasible } \\
\text { with force applied during } \\
\text { process }\end{array}$ & $\begin{array}{l}\text { Good } \\
\text { no deformation and good } \\
\text { performance }\end{array}$ & $\begin{array}{l}\text { Feasible } \\
\text { with force applied during } \\
\text { process }\end{array}$ \\
\hline Laser welding & $\begin{array}{l}\text { Feasible } \\
\text { but spot welding only }\end{array}$ & $\begin{array}{l}\text { Feasible } \\
\text { but spot welding only }\end{array}$ & Not feasible \\
\hline EB welding & $\begin{array}{l}\text { Very good } \\
\text { no thermal resistance } \\
\text { between foils }\end{array}$ & $\begin{array}{l}\text { Very good } \\
\text { no thermal resistance } \\
\text { between foils }\end{array}$ & Not feasible \\
\hline Brazing & $\begin{array}{l}\text { Good } \\
\text { with additional thermal } \\
\text { resistance }\end{array}$ & $\begin{array}{l}\text { Good } \\
\text { with additional thermal } \\
\text { resistance }\end{array}$ & $\begin{array}{l}\text { Good } \\
\text { with additional thermal } \\
\text { resistance }\end{array}$ \\
\hline Swaging & $\begin{array}{l}\text { Good } \\
\text { with additional contact } \\
\text { resistance between foils }\end{array}$ & $\begin{array}{l}\text { Good } \\
\text { with additional contact } \\
\text { resistance between foils }\end{array}$ & $\begin{array}{l}\text { Good } \\
\text { with additional contact } \\
\text { resistance between foils }\end{array}$ \\
\hline
\end{tabular}

图 13 复合柔性石墨冷链（左图）和一体式柔性石墨冷链（右图）

Fig.13 Compound graphite flexible thermal link (left) and integrated graphite flexible thermal link (right)

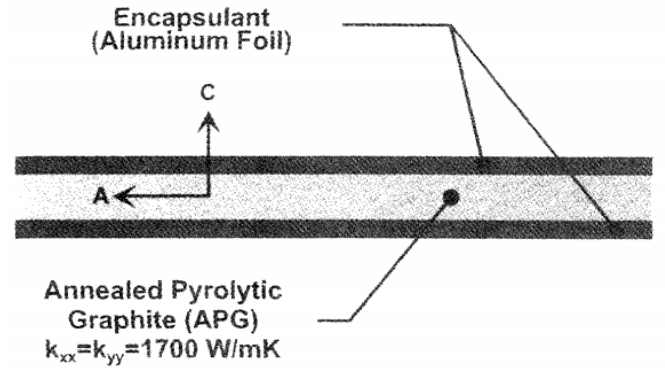

图 14 热解石墨柔性冷链结构

Fig.14 Flexible thermal link structure of APG
美国的 Akira Yao 提出对碳纤维纸柔性冷链结构 减小温度梯度的解决方法，如图 15 所示。可通过在 冷链热接口两端, 在碳纤维纸之间夹入金属薄片来均 匀由于碳纤维纸各向异性热导率带来的温度梯度 ${ }^{[10]}$ 。

\section{2 柔性冷链的国内研究现状}

随着我国在空间领域的不断发展, 对各种空间应 用探测器的要求与性能不断提升, 由于柔性冷链在隔 绝机械制冷机带来的振动和灵活传输以及分配冷量 方面具有很好的效果, 我国的研究人员也对柔性冷链 
进行了研究。

国内主要以紫铜丝导热带或多片铜薄片组来制成 柔性冷链。2007 年, 中国科学院理化研究所采用 3000 根直径为 $0.01 \mathrm{~cm}$ 紫铜丝绞在一起的紫铜丝导热带制 作成柔性导热带用于整机结构中 ${ }^{[11]}$; 2013 年, 兰州空 间技术物理研究所的孙述泽采用了 30 片 $0.2 \mathrm{~mm}$ 厚度 长 $100 \mathrm{~mm}$ 紫铜䈃弯曲成双臂状制作成柔性导热用于 高温超导滤波器的降温 ${ }^{[12]}$; 中国上海技物所在多个项 目中使用紫铜薄片组成的柔性冷链为其提供冷链传 输, 主要应用在同一制冷源为多种组件提供制冷, 多 台制冷机为一个组件提供制冷和超长线列红外探测器 等应用上, 以 2015 年该所的范广宇提出的一种弹性冷 链的设计方案为例, 为满足超长线列红外探测器杜瓦 组件的需求, 该冷链设计为采用多片厚 $0.25 \mathrm{~mm}$, 弯曲 成 S 形, 沿水平方向切割成四等分的铜薄片来作为柔 性结构, 通过对热接口位置及各连接处不同的厚度进 行模拟计算, 使各个分枝的热阻相同, 来满足杜瓦冷 平台温度均匀性要求, 该冷链最终实测结果为其冷平 台在液氮温度下最大温差为 $1.6 \mathrm{~K}$, 冷链上下两端导 热温差为 $6.2 \mathrm{~K}^{[2]}$, 该冷链结构如图 16 所示。

\section{3 冷链性能测试}

柔性冷链的性能主要是集中在力学和热学方面,

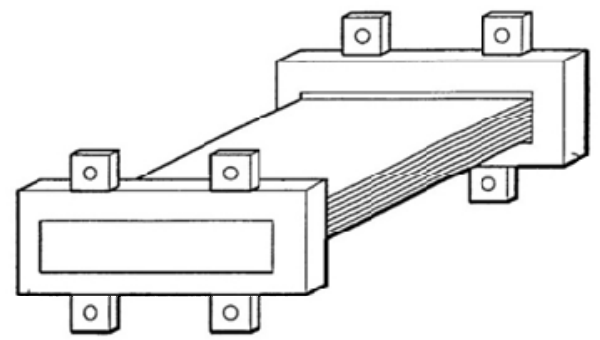

可细分别为以下几个性能指标:

1) 柔性冷链整体本身的热导率或热阻;

2）柔性冷链的各个方向的刚度;

3）柔性冷链的隔振效果;

4）柔性冷链的疲劳特性。

国外能够对冷链的热阻、刚度、隔振效果和疲劳 特性进行测量, 给出柔性冷链完整的指标性能。如图 17 所示为柔性冷链热学性能测试平台, 将冷链处于真 空环境中来减小对流漏热的影响, 通过防辐射屏或包 裹镀铝聚酰亚胺薄膜来减小辐射漏热的影响, 使用热 电阻或者加热片来模拟热耗确定热流, 液氮或者制冷 机进行制冷, 温度传感器测量冷链上下两端的温度, 便可计算出冷链整体的热导率或热阻 ${ }^{[13]}$ 。

如图 17 所示, 通过测量冷链各个方向的应变随应 力的变化关系可测得冷链各个方向的刚度 ${ }^{[8]}$; 在振动台 或振动源上, 通过振动加速度传感器测量冷链两端的 振动, 可计算出冷链的隔振性能 ${ }^{[14]}$; 通过在冷链上加 载相应正弦周期变化的力, 可测得冷链的疲劳性能。

国内也对冷链的测量进行相关的研究工作, 主要 通过将冷链装载在应用部件内对冷链的部分热学性 能进行了测量 ${ }^{[15]}$, 如冷链上下两端的温差等热学性能 参数, 但很少对冷链的力学性能如刚度、隔振和疲劳 特性等参数进行测量。

图 15 碳纤维纸柔性冷链结构 (左图) 和冷链夹层结构 (右图)

Fig.15 Flexible thermal link structure of carbon fiber paper (left) and sandwich structure of thermal link (right)
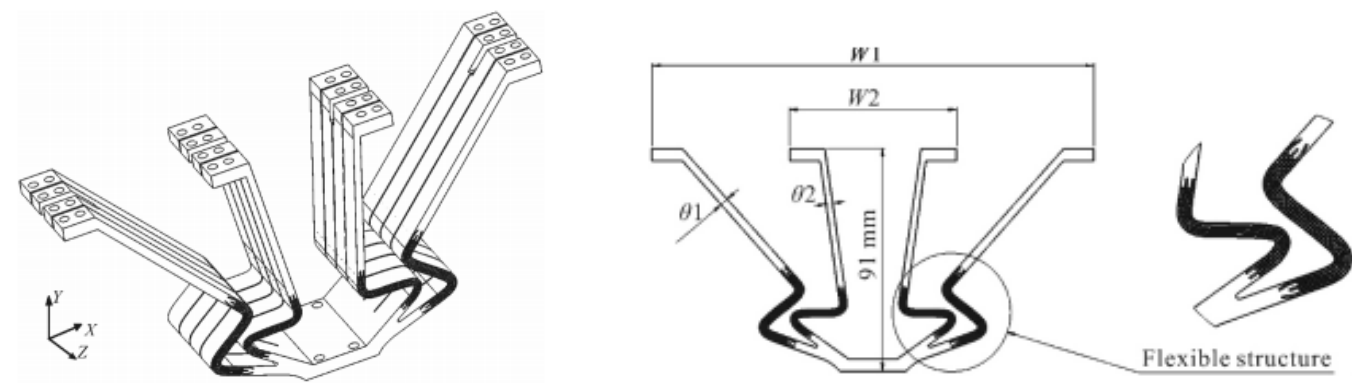

图 16 直线脉管集成耦合杜瓦柔性冷链结构

Fig.16 Flexible thermal link structure of linear pulse tube cooler dewar assembly 


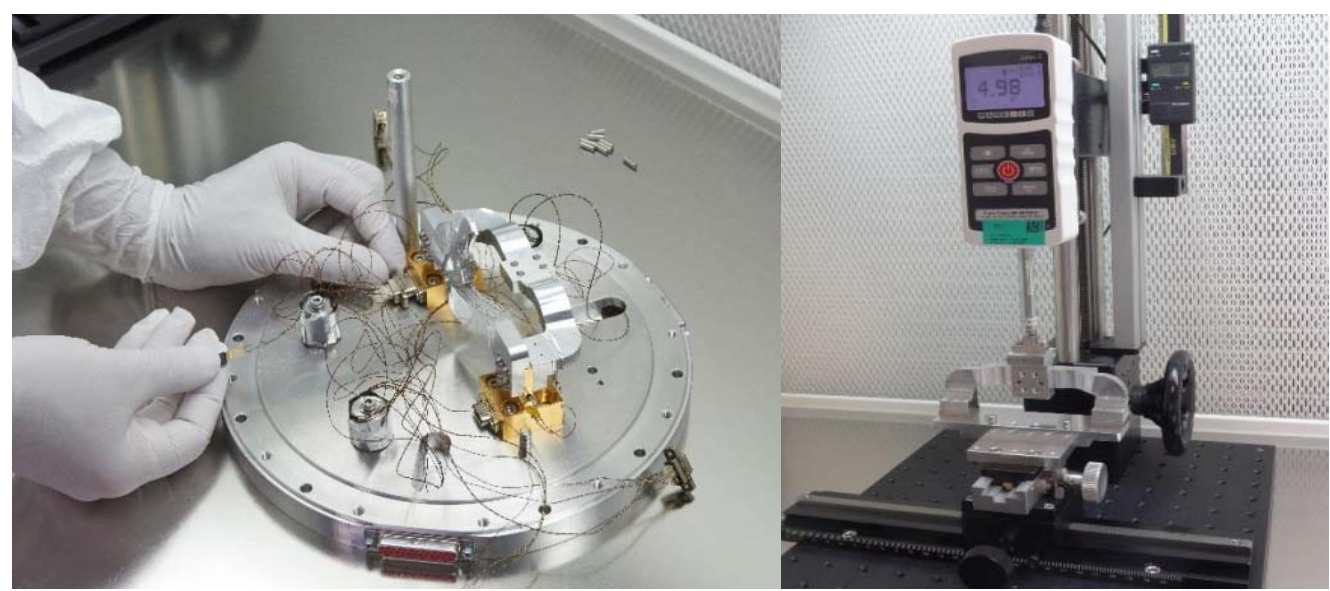

图 17 冷链热学性能测试平台 (左图) 和冷链刚度测试平台 (右图)

Fig.17 Thermal performance test platform of thermal link(left) and stiffness test platform of thermal link(right)

\section{4 应用及展望}

随着三代红外焦平面探测器组件更大面阵、双多 色的发展, 和我国航天事业的飞速发展, 大面阵、长 线列红外探测器和拼接式红外探测器将大量应用于 航天领域, 新型的探测器杜瓦与制冷机的耦合方式将 逐渐普及, 本文提及的各种柔性冷链的设计、制作和 测试也将会逐渐完善, 在未来超长线列红外探测器与 制冷机之间的耦合技术中将全方面发展。

\section{参考文献:}

[1] 东海杰, 张否, 白绍竣, 等. 长线列红外探测器组件冷台面结构设计 [J]. 激光与红外, 2018, 48(8): 1020-1022.

DONG Haijie, ZHANG Lei, BAI Shaojun, et al. Structure design of the cold table for long linear infrared detector assembly[J]. Laser \& Infrared, 2018, 48(8): 1020-1022.

[2] 范广宇, 范崔, 李俊, 等. 超长线列红外焦平面杜瓦冷链设计[J]. 红外 与激光工程, 2015, 44(7): 2021-2016.

FAN Guangyu, FAN Cui, LI Jun, et al. Cold strap design of long linear IRFPA dewar[J]. Infrared and Laser Engineering, 2015, 44(7): 2021-2016.

[3] Dolce S, Hauser A. Analysis, design and testing of a flexible thermal link[J]. Space Environmental Control Systems, 1997: 337-340.

[4] Amundsen M E. SAE Technical Paper Series - Thermal Design and Analysis for the Cryogenic MIDAS Experiment[C]/SAE International Conference On Environmental Systems, 1997: 1.

[5] LI J, Schmit $\mathrm{T}$ J. Advanced baseline sounder (ABS) for future geostationary operational environmental satellites (GOES-R and beyond)[C]//Proceedings of SPIE-The International Society for Optical Engineering, 2003, 4895: 111-122.

[6] Morse P G, Bates J C, Miller C R, et al. Development and test of the Atmospheric Infrared Sounder (AIRS) for the NASA Earth Observing System (EOS)[C]//Sensors, Systems, \& Next-generation Satellites III. Sensors, Systems, and Next-Generation Satellites III, 1999: 281-292.
[7] Sugimoto M, Sekimoto Y, Yokogawa S, et al. Thermal link for cartridge-type cryostat[J]. Cryogenics, 2003, 43(8): 435-439.

[8] Trollier T, Tanchon J, Lacapere J, et al. Flexible Thermal Link Assembly Solutions for Space Applications[J]. Cryocoolers, 2016, 19: 595-603.

[9] Montesano M. High erformance flexible thermal strap[C]/39th Aerospace Sciences Meeting and Exhibit, 2001: 218.

[10] Yao A. Interface portion structure and reinforcing structure of flexible thermal joint: U.S. Patent 5,769,158[P]. 1998-6-23.

[11] 间涛, 洪国同, 蔡京辉, 等. 一种应用于低温系统的柔性冷量传输带 [C]//全国低温工程大会暨中国航天低温专业信息网学术交流会, 2007: 299-303.

YAN Tao, HONG Guotong, CAI Jinghui, et al. A flexible thermal strap applied to cryogenic system[C]/National Cryogenic Engineering Conference and Acedemic Meeting of China Aerospace Cryogenic Information Network, 2007: 299-303.

[12] 孙述泽, 许国太, 王田刚, 等. 一种双臂型低温导热带的性能研究 [J]. 低温与超导, 2013, 41(5):10-12.

SUN Shuze, XU Guotai, WANG Tiangang, et al. Study on performance of cryogenic two-arm flexible thermal strap[J]. Cryogenics, 2013, 41(5): $10-12$.

[13] Urquiza E, Vasquez C, Rodriguez J, et al. Development and testing of an innovative two-arm focal-plane thermal strap(TAFTS)[J]. Cryogenics, 2012, 52(4-6): 0-3.

[14] Williams B, Jensen S, Batty J C. An Advanced Solderless Flexible Thermal Link[C]//Proc of SPIE, 1996, 2814: 209-216.

[15] 王亚妮, 张巍, 迟国春, 等. 制冷机与红外探测器冷链耦合技术研究 [J]. 激光与红外, 2018, 48(2): 148-152.

WANG Yani, ZHANG Wei, CHI Guochun, et al. Research of cold chain linking technology between cryocooler and infrared detector[J]. Laser \& Infrared, 2018, 48(2): 148-152. 